\title{
The Structure of Two-dimensionally Isotropic Turbulence*
}

\author{
Y. Ogura \\ (Geophysical Institute, Tokyo University)
}

\begin{abstract}
- Abstract -
Considering that many atmospheric phenomena can well be regarded to be nearly two-dimensional, the author theoretically daals with the two-dimensionally isotropic turbulent field. The structure of the field is investigated in close connection with that of the three-dimensionally isotropic turbulent field. Taylor-Kármán's correlation coefficient for the former is found to be approximately the same with that for the latter in their numerical values, though their functional forms are considerably different from each other. The calculated numerical values of the coefficient agree well with those actually observed in the atmosphere.
\end{abstract}

\section{§ 1. Introduction.}

In many meteorological phenomena occurring in the atmosphere, the horizontal dimensions of the phenomena are so much larger than the vertical one, that the motion of the air participating in the phenomena can well be regarded to be two-dimensional. It may be reasonably assumed that the two-dimensionally isotropic turbulence is effective for these phenomena, rather than the three-dimensionally isotropic. For instance, cyclones and anticyclones, which may be considered as effective eddies for the global metcorological phenomena, have the horizontal dimensions of the order of $1,000 \mathrm{~km}$ and the vertical one of only $10 \mathrm{~km}$. Diffusion phenomena of ashes from volcanoes in eruption may be controlled also by the two-dimensional turbulence. When we study the micro-structures of winds in the lowest layer of the atmosphere, the turburence detected can bo regarded two-dimensional also, for, if the mean wind velocity during an observation is $5 \mathrm{~m} / \mathrm{sec}$, and the timeduration of the observation is $10 \mathrm{~min}$., the horizontal scale of turbulence is $3 \mathrm{~km}$, while the vertical one is of the order of the height of the observation point and is small.

Since 1940, a remarkable progress has been made in the theory of isotropic turbulence and the observed facts in wind tunnels have confirmed the validity of the theory.

Recently, the similarity theory was successfully applied to the atmospheric turbulence. In these applications, however, the theory of three-dimensionally isotropic turbulence was used in its original form. From the reason stated at the beginning of the paper, it is desirable to study the structure of the two-dimensionally isotropic turbulence and to make clear the difference between the energy spectrum functions (or Taylor-Kármán's correlation coefficients) for three- and two-dimensionally isotropic turbulences. In the present paper, this problem will be studied and the theoretical results will be compared with the observed.

* Division of Meteorology, Contribution No. 43 . 


\section{$\S 2$. The kinematics of two-dimensionally isotropic turbulence.}

First of all, we shall see the interrelation between Taylor-Kármán's correlation coefficients of turbulent wind velocities, following Th. von Kármán and L. Howarth (1987).

Consider two points $P$ and $Q$ in a fluid. Denote by $p$ the velocity component in a particular direction $P P^{\prime}$ at $P$ and by $q$ the velocity component in the direction $Q Q^{\prime}$ at $Q$. Moreover, denote by $\alpha, \beta$ the angles $\widehat{P^{\prime} P Q}, \pi-\widehat{P Q Q}$, and by $p_{1}, p_{2}$ and $q_{1}$, $q_{2}$, respectively, the velocity components at $P$ and $Q$ in the direction $P Q$ and the direction normal to $P Q$ (Figure 1). Then

$$
\begin{aligned}
& p=p_{1} \cos \alpha+p_{2} \sin \alpha, \\
& q=q_{1} \cos \beta+q_{2} \sin \beta .
\end{aligned}
$$

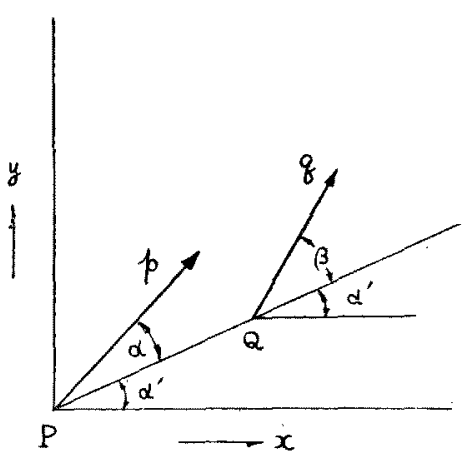

Fig. 1

$\langle p q\rangle^{*}=\left\langle p_{1} q_{1}>\cos \alpha \cos \beta+<p_{1} q_{2}>\cos \alpha \sin \beta+<p_{2} q_{1}>\sin \alpha \cos \beta+<p_{2} q_{2}>\sin \alpha \sin \beta\right.$. From the assumption that turbulence is isotropic, $\left\langle p_{1} q_{2}\right\rangle$ and $\left\langle p_{2} q_{1}\right\rangle$ vanish. Thus, we obtain the following relation:

$$
\frac{<p q>}{<u^{2}>}=f(r, t) \cos \alpha \cos \beta+g^{\prime}(r, t) \sin \alpha \sin \beta
$$

where $f(r, t)$ and $g(r, t)$ denote the correlation coeficients $\left\langle p_{1} q_{1}\right\rangle \mid\left\langle u^{2}\right\rangle$ and $\left\langle p_{2} q_{2}\right\rangle \mid$ $\left\langle u^{2}\right\rangle, r$ the distance between $P$ and $Q$. Consider now a particular co-ordinate system, and denote the velocity components in that system at $P\left(x_{1}, x_{2}\right)$ and $Q\left(x_{1}{ }^{\prime}, x_{2}{ }^{1}\right)$ by $\left(u_{1}, u_{2}\right)$ and $\left(u_{1}^{\prime}, u_{2}{ }^{\prime}\right)$, respectively. Then the correlation tensor $\boldsymbol{R}$ is

$$
<u^{2}>\boldsymbol{R}=\left\langle u^{2}>\left(\begin{array}{l}
R_{11} R_{12} \\
R_{21} R_{23}
\end{array}\right)=\left(\begin{array}{l}
<u_{1} u_{1}^{\prime}><u_{1} u_{2}^{\prime}> \\
<u_{4} u_{1}^{\prime}><u_{2} u_{2}^{\prime}>
\end{array}\right),\right.
$$

or

$$
\left\langle u^{2}\right\rangle \boldsymbol{R}=\left\langle u^{2}\right\rangle R_{i j}=\left\langle u_{i} u_{j}{ }^{\prime}\right\rangle . \quad i=1 \text { or } 2 .
$$

By means of $(2 \cdot 1), \boldsymbol{R}$ can be evaluated in terms of the functions $f$ and $g$ and of the vector whose components are $\xi_{1}=x_{1}^{\prime}-x_{1}, \xi_{2}=x_{2}^{\prime}-x_{2}$. In this way we obtain

$$
\boldsymbol{R}=\frac{\{f(r, t)-g(r, t)\}}{r^{2}} \boldsymbol{r} \boldsymbol{r}+g(r, t) \boldsymbol{I},
$$

where $r=|\boldsymbol{P}|$ and $\boldsymbol{I}$ is the unit tensor

$$
\left(\begin{array}{ll}
1 & 0 \\
0 & 1
\end{array}\right)
$$

Now, the velocity fluctuations at $Q$ satisfy the equation of continuity:

$$
\frac{\partial u_{i}^{\prime}}{\partial x_{i}^{\prime}}=0
$$

* The set of symbols $<>$ means to take average over an appropriate domain. 
where the summation convention is in operation. Therefore

$$
\frac{\partial}{\partial x_{i}{ }^{\prime}}\left(u_{i}^{\prime} u_{j}\right)=0
$$

since $u_{r}$ is independent of $x_{l}{ }^{\prime}$. Thus we obtain

$$
\text { or } \quad \begin{aligned}
\frac{\partial R_{s i}}{\partial x_{i}} & =0, \\
\frac{\partial R_{j i}}{\partial \xi_{i}} & =0 .
\end{aligned}
$$

Using the values of the components of $\boldsymbol{R}$ given by equation $(2 \cdot 2)$, we find

$$
g(r, t)=f(r, t)+r \frac{\partial f(r, t)}{\partial r} .
$$

This equations corresponds to

$$
g(r, t)=f(r, t)+\frac{r}{2} \frac{\partial f(r, t)}{\partial r},
$$

which holds for the three-dimensionally isotropic turbulence.

\section{$\S 3$. Energy Spectra.}

We shall assume that the two components of the velocity in a homogeneous isotropic turbulent field can be developed, at any instance, by Fourier's integral:

$$
u_{i}\left(x_{1}, x_{i}, t\right)=\frac{1}{2 \pi} \iint_{-\infty}^{\infty} \Phi_{i}\left(k_{1}, k_{i}, t\right) e^{i\left(k_{2} x_{2}+k_{2}, x_{2}\right)} d k_{1} d k_{i},
$$

where $k_{1}$ and $k_{z}$ denote the wave numbers along the $x$ - and $y$-axes respectively.

By averaging $(3 \cdot 1)$ over a sulficiently large area $s$, we get

$$
\left\langle u_{i}^{2}\right\rangle=\frac{1}{s} \iint_{-\infty}^{\infty}\left|\Phi_{i}\right|^{2} d k_{1} d k_{\ldots} \text {. }
$$

Then, we have

$$
\left\langle u_{1}^{2}\right\rangle=\frac{1}{2 S} \int_{-\infty}^{\infty}\left\{\left|\mathscr{Q}_{1}\right|^{2}+\left|\mathscr{Q}_{2}\right|^{2}\right\} d k_{1} d k_{\varkappa_{2}}
$$

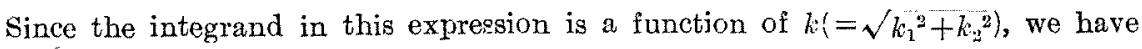

$$
\left.<u^{2}\right\rangle=\int_{0}^{\infty}<u^{2}>F_{*}(k) d k
$$

where

$$
\left\langle u^{2}>F_{*}(k)=\frac{\pi}{S} k\left\{\left|\Phi_{1}\right|^{2}+\left|\emptyset_{:}\right|^{2}\right\}\right.
$$

Iet $F\left(k_{1}\right)$ and $G\left(k_{1}\right)$ denote the spectral functions for the energies passing through a fixed cross section in a turbulent stream, we get from $(3 \cdot 1)$. 


$$
\begin{aligned}
\left\langle u_{1}^{2}\right\rangle & =\int_{0}^{\infty}\left\langle u^{2}\right\rangle F\left(k_{1}\right) d k_{1}=\frac{1}{S} \int_{-\infty}^{\infty} d k_{1} \int_{-\infty}^{\infty}\left|\Phi_{1}\right|^{2} d k_{\cdot \cdot \cdot} \cdot \\
\text { and } \quad & \left\langle u_{2}^{2}\right\rangle=\int_{0}^{\infty}\left\langle u^{2}\right\rangle G\left(k_{1}\right) d k_{1}=\frac{1}{S} \int_{-\infty}^{\infty} d k_{1} \int_{-\infty}^{\infty}\left|\Phi_{2}\right|^{2} d k_{2 \cdot} .
\end{aligned}
$$

By summing up $(3 \cdot 5)$ and $(3 \cdot 6)$ and referring to $(3 \cdot 4)$, we have

$$
F\left(k_{1}\right)+G\left(k_{1}\right)=\frac{2}{\left.S<u^{2}\right\rangle} \int_{-\infty}^{\infty}\left\{\left|\Phi_{1}\right|^{2}+\left|\Phi_{2}\right|^{2}\right\} d k_{2}=\frac{1}{\pi} \int_{k_{1}}^{\infty} \frac{F_{*}(k)}{\sqrt{k^{2}-k_{2}{ }^{2}}} d k
$$

Now, the spectral functions $F\left(k_{1}\right)$ and $G\left(k_{1}\right)$ are related to the correlation functions $f(x)$ and $g(x)$ as follows:

$$
\left.\begin{array}{ll}
f(x)=\int_{0}^{\infty} F\left(k_{1}\right) \cos k_{1} x d k_{1}, & F\left(k_{1}\right)=\frac{2}{\pi} \int_{0}^{\infty} f(x) \cos k_{1} x d x . \\
g(x)=\int_{0}^{\infty} G\left(k_{1}\right) \cos k_{1} x d k_{1}, & G\left(k_{1}\right)=\frac{2}{\pi} \int_{0}^{\infty} g(x) \cos k_{1} x d x .
\end{array}\right\}
$$

Then by the relation $(2 \cdot 3)$ we get the following interrelation between $F\left(k_{1}\right)$ and $G(k)$ :

$$
G\left(k_{1}\right)=-k_{1} \frac{d F\left(k_{1}\right)}{d k_{1}}
$$

From $(3 \cdot 9)$ and $(3 \cdot 7)$ we get finally

$$
F\left(k_{1}\right)=\frac{2}{\pi} \int_{k_{1}}^{\infty} F_{*}(k) \frac{\sqrt{k^{2}-k_{1}^{2}}}{k^{2}} d k .
$$

This relation corresponds to the equation obtained by Heisenberg (1948) for the case of three-dimensionally isotropic turbulence:

$$
F\left(k_{1}\right)=\frac{3}{2} \int_{k_{1}}^{\infty} F_{*}(k) \frac{k^{2}-k_{1}^{2}}{k^{3}} d k .
$$

\section{$\S$ 4. Numerical evaluation.}

The spectrum of energy for the three-dimensionally isotropic turbulence has been derived by many authors. For instance, in 1948, Th. von Kármán obtained it in the following form:

$$
F_{*}\left(\frac{k}{k_{0}}\right) \sim \frac{\left(\frac{k}{k_{n}}\right)^{4}}{\left\{1+\left(\frac{k}{k_{0}}\right)^{2}\right\}^{17 / 6}}
$$

It can easily be proved that this energy spectrum function is valid also for the two-dimensionally isotropic turbulence, if we follow the same procedure that was taken by Kármán to derive this result. By substituting $(4 \cdot 1)$ into $(3 \cdot 10)$ and integrating numerically, we 
can get $F\left(k_{1}\right)$. The dotted line in Figure 2 shows this numerically evaluated result. For comparison, $F\left(k_{\mathrm{t}}\right)$ for the three-dimensionally isotropic turbulence expressed by $(4.2)$ is also shown in this figure by the full line:

$$
F\left(k_{1}\right)=\text { const. } \frac{1}{\left\{1+\left(\frac{k_{1}}{k_{0}}\right)^{2}\right\}^{5 / 6}}
$$

The constant values in $F\left(k_{1}\right)$ for both cases are determined by the condition

$$
\int_{0}^{\infty} F\left(k_{1}\right) d k_{1}=1
$$

Then, substituting $F\left(k_{1}\right)$ into $(3 \cdot 8)$, TaylorKármán's correlation coeffiicient $f(x)$ can be

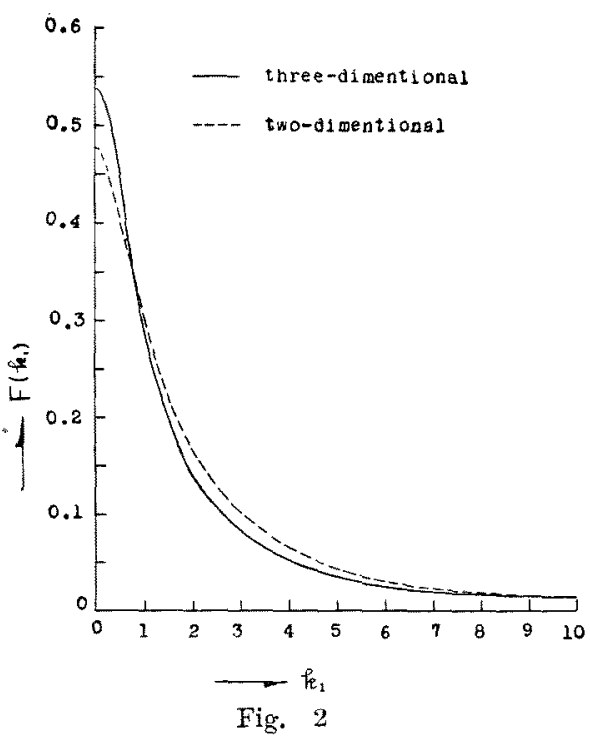
evaluated. In Figure 3 the dotted and full lines show these $f(x)$, both for the two- and three-dimensionally isotropic turbulences. $f(x)$ for the latter is expressed by $(4 \cdot 2)$ and $(3 \cdot 8)$ as follows:

$$
f\left(k_{0} x\right)=\frac{2^{2 / 3}}{\Gamma(1 / 3)}\left(k_{0} x\right)^{T / 3} K_{1 / 3}\left(k_{0} x\right)
$$

wheer $K_{1 / 3}$ is a Bessel function with imaginary argument.

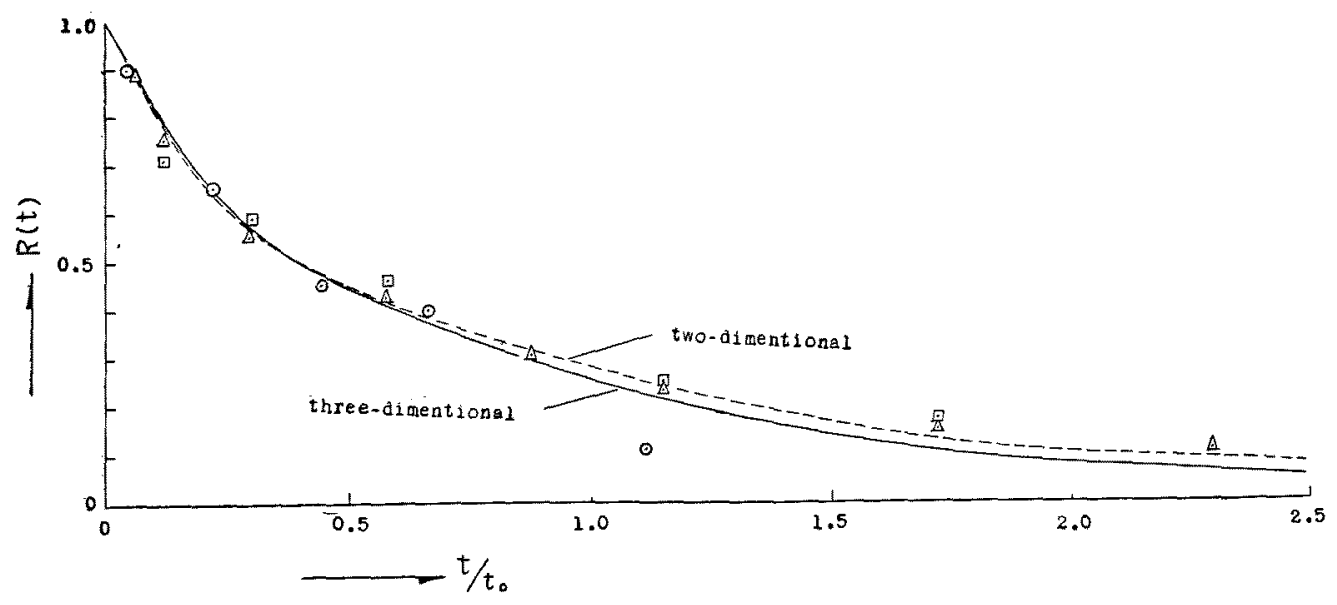

Fig. 3

Points in this figure represent the values which were obtained experimentally by $G$. Yamamoto and M. Shiotani (1948) by means of a hot-wire anemometer at 38 meters above 
the ground. As it can be seen easily, these two curves agree approximately with each other, though their functional forms are much different, and both curves agree fairly well with the observed data. According to this result, it may reasonably said that no serious error is caused even if the functional form of $f(x)$ of three-dimensionally isotropic turbulence is used in order to study the two-dimensional motion of air.

In his previous papers, the author put forward a theory on the relationship between the length of time of analysis and the statistical quantities of turbulence. In comparing these theoretical results with observed data obtained in the lowest layer of the atmosphere, he made use of the functional form of $(4.4)$, which hold only for the case of three-dimensionally isotropic turbulence, and a fairly good agreement between the theoretical and observational results was obtained $(1950,1952 \mathrm{a})$. The physical bases of this agreement are to bo found in the considerations albove mentioned.

Moreover, the author treated theoretically the turbulent diffusion in the atmosphere in other papers $\left(195^{2} \mathrm{~b}, \mathrm{c}_{i}\right.$. Though the results were derived assuming that the turoulence was three-dimensionally isotropic, they hold approximately good for the cases of horizontal diffusion, also.

In conclusion the author wishes to express his sincere thanks to Prof. Syono for his. kind encouragement and guidance.

\section{References}

Heisenberg, W. 1948: Zur statistischen Theorie der Turbulenz. Zeits. f. Phys., 194, Heft 7/12, ss. $628-659$.

Kármán, Th, von and Howarth, L. 1938: On the Statistical Theory of Isotropic Turbulence. Proc. Roy. Soc. London, 164, pp. 192-215.

Kármán, Th. von, 1948: Progress in the Statistical Theory of Turbulence. Proc. Nat. Acad. Science, 34, No. 11, pp. 530-539.

Ogura, Y. 1950: On the Atmospheric Turbulence. Geophys. Notes, 3, No. 26. 1952 a: The Relations between the Statistical Quantities of Atmospheric Turbulences and the Length of Time of Analysis. Journ. Met. Soc. Japan, in the press.

1952 b: The Theary of Turbulent Diffusion in the Atmosplese I. Journ. Met. Soc. Japan, 30, 53.

- 1952 c: The Theory of Turbulent Diffusion in the Atmosphere II. Journ. Met. Soc. Japan, 30, 53.

Taylor, G. I. 1938: The Spectrum of Turbulence. Proc. Roy. Soc. London, 164, p. 476.

Yamamoto, G. and M. Shiotani 1948: Turbulence in the Free Atmosphere (2nd Report). Journ. Met. Soc. Japan, 26, pp. 149-157. 